\title{
INFLUÊNCIA DO ALONGAMENTO NO ESTRESSE PRÉ-COMPETITIVO EM JOGADORES DE FUTEBOL DA CATEGORIA JUVENIL
}

\author{
STRETCH INFLUENCE ON PRE-COMPETITIVE STRESS \\ ON JUVENILE SOCCER PLAYERS
}

\author{
AUTORES \\ José Alexandre Curiacos de Almeida Leme' \\ Ricardo Augusto Barberi ${ }^{2}$ \\ Karina Junqueira Curiacos ${ }^{3}$ \\ Priscila Valim Rogatto ${ }^{4}$ \\ ${ }^{1}$ Mestrando - Departamento de Educação \\ Física, UNESP - Rio Claro, Brasil \\ ${ }^{2}$ Graduando - Departamento de Educação \\ Física, UNESP - Rio Claro, Brasil \\ ${ }^{3}$ Graduada - Faculdade de Psicologia, \\ Unimep- Piracicaba, São Paulo, Brasil \\ ${ }^{4}$ Docente - Departamento de Educação Física, \\ UFMG - Cuiabá - Mato Grosso, Brasil
}

INFLUÊNCIA DO ALONGAMENTO NO ESTRESSE PRÉ-COMPETITIVO EM JOGADORES DE FUTEBOL DA CATEGORIA JUVENIL 4(3): 57-60

\section{PALAVRAS-CHAVE}

alongamento; estresse; futebol.

\section{KEYWORDS}

stretching; stress; soccer.

\section{RESUMO}

0 futebol apresenta uma séria de situações geradoras de estresse. 0 momento pré-competitivo é altamente gerador de estresse. 0 alongamento pode contribuir para diminuir estes efeitos negativos do estresse. 0 objetivo deste estudo foi verificar os efeitos do alongamento no estresse pré-competitivo de jogadores de futebol. Participaram deste estudo 17 atletas juvenis, distribuídos em dois grupos: Grupo Controle (GC) e Grupo Alongamento (GA). Todos responderam ao TEF e, em seguida, o GA praticou 30 minutos de alongamento enquanto o GC vivenciou as situações habituais sem realização de atividade física. Logo após, os dois grupos responderam novamente ao TEF, sendo invertidos os grupos na partida seguinte. As situações "Dormir mal a noite anterior ao jogo" e "Ser prejudicado pelos juizes" foram avaliadas com respostas mais negativas enquanto "Cobrança de si mesmo para ganhar" e "O time adversário é o favorito" foram avaliadas como respostas mais positivas. 0 GA obteve aumento no número de avaliações positivas comparado ao GC, diminuição no nulo e diminuição na resposta -1 e -3, enquanto o GC obteve diminuição no valor -2 . Conclui-se que a situação pré-competitiva modifica negativamente a avaliação dos atletas enquanto $o$ alongamento interfere positivamente frente à avaliação das situações estressoras em jogadores juvenis.
ABSTRACT

Soccer show a series of stress-generated situations. The streatching may contribut to decrease this stress negative effects. The pre-competitive moment is stress generator. The objetive of this study was to verify the effects of strectch on pre-competitive stress in soccer players. To this, 17 juvenile soccer players were allocated into two groups: control group (CG) and strecth group [SG]. All participants responsed to TEF (Stress soccer test) and GA stretching during $\mathbf{3 0}$ minutes while GC not realized any physical activity. After this, both groups responded to TEF and was inverted in the next game. The situations "sleep badly on previous night" and "Be damaged for arbitrator" was avaliated with more negative reponses while "Himself charged to win" and "The adversary is the favourite" was avaliated with more positive reponses. SG showed positive avaliations increased copared to CG, decreased on nule response and decreased on -1 and -3 responses. GC showed decrease in -2 responses. Concluded that pre-competitive situation modify negatively the athletes avaliation while the strecth interfere positively in front the avaliation of stressor situations in juvenile players. 


\section{INTRODUC̣ÃO}

0 futebol é considerado, no Brasil, o esporte mais popular e praticado e leva multidões aos estádios, mexendo com o cotidiano de boa parte da população. Muitos jovens aderem ao futebol com a expectativa de se tornarem atletas de sucesso nacional e internacional. Poucos são os que conseguem chegar ao profissionalismo, porque para isto são fundamentais alguns pré-requisitos, dentre eles a aptidão física, habilidades técnico-táticas e competência emocional ${ }^{1}$.

Segundo Samulski e Chagas ${ }^{2}$, os jogadores de futebol estão expostos a um número potencial de estressores que operam sobre o desempenho atlético durante a carreira. Têm sucesso aqueles que conseguem sobreviver as enormes pressões do esporte e que superam angustias e incertezas. Os jogadores de categorias amadoras que se preparam para o profissionalismo se encontram em fases ainda mais sensiveis de desenvolvimento da personalidade e podem apresentar maiores dificuldades no controle de suas emoções e reações antes, durante ou depois das competições, apesar de a agressividade aumentar com o aumento da idade em jogadores de futebol ${ }^{2}$. Por isso necessitam de um suporte psíquico e social que os sustente para desenvolverem todas as habilidades requeridas pela modalidade esportiva. Um dos fatores de natureza psicológica que distingue o atleta vencedor de um perdedor é sua capacidade de lidar com o estresse da competição'.

De acordo com Samulski e Chagas ${ }^{2}$ algumas situações esportivas podem desencadear o distress causando três tipos de sintomas negativos: físico, mental e comportamental. Porém existe outra forma de reação estressora considerada positiva, o eustress que deixa o atleta motivado psicologicamente e estimulado fisiologicamente, preparando o organismo para a performance máxima.

0 agente estressor nem sempre é negativo, podendo desencadear reações adaptativas do sistema nervoso interpretadas pelo organismo sem distinção. A maneira como cada pessoa sente e percebe o mesmo agente estressor faz com que o estresse seja caracterizado como positivo ou negativo ${ }^{4}$.

Estudos prévios tem demonstrado que exercício físico tem geralmente apresentado que a atividade física está associada à redução dos efeitos negativos do estresse ${ }^{5,6 .}$ Após suas pesquisas, Valim ${ }^{4}$ comprovou que um alongamento específico reduz significativamente o número de sintomas [psicológicos e físicos) do estresse em jovens que se encontravam em uma determinada situação estressora.

0 momento pré-competitivo é uma situação altamente geradora de estresse para o atleta ${ }^{7}$. Os objetivos do presente estudo foram: a) verificar se uma série de $\mathbf{3 0}$ minutos de alongamento pode interferir no estresse de jogadores de futebol de campo, que estejam em fase de competição e b] Se a referida interferência ocorrer, observar se é positiva ou negativa para o atleta.

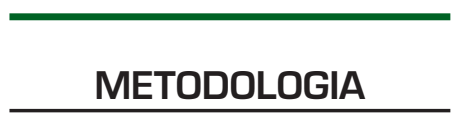

\section{Amostra}

A pesquisa foi realizada com 17 jogadores de futebol de campo da categoria juvenil (15 a 17 anos) de duas equipes, de Piracicaba e Rio Claro, ambas do estado de São Paulo, e sendo todos jogadores de linha e titulares. As coletas foram feitas durante jogos do campeonato paulista e do campeonato municipal de Rio Claro.

\section{Instrumentos}

Para a avaliação do estresse foi utilizado o Teste de Estresse no Futebol - TEF $^{2}$ (anexo 1). Este instrumento contém 30 situações de jogo onde o atleta avalia a influência de cada situação como positiva, nula ou negativa. A análise é faita baseado numa escala de valores de sete categorias (de +3 a -3). A série de alongamentos aplicados foi proposta por Valim ${ }^{4}$ para situações estressoras.

\section{Procedimentos}

Os dados foram coletados em duas partidas. Em ambas, no período de uma hora antes do inicio da partida, os atletas foram aleatoriamente distribuídos em dois grupo: Grupo Controle (GC) e Grupo Alongamento [GA].

Primeiramente os dois grupos responderam ao TEF. Em seguida, o GA praticou os 30 minutos do alongamento proposto por Valim ${ }^{4}$ enquanto o GC vivenciou as situações habituais de espera de jogo ainda sem realização de nenhuma atividade física pré-competitiva. Logo após este período, os dois grupos responderam novamente ao TEF.

Na partida seguinte, os grupos foram invertidos, sendo que os atletas que participaram do GA passaram a fazer parte do GC e vice-versa.

\section{Análise estatística}

Foram computadas as médias de cada fator antes e após as situações experimentais para verificar possíveis alterações.

\section{RESULTADOS}

A figura 1 apresenta as médias das avaliações feitas pelos atletas nas determinadas situações. As situações "Dormir mal a noite anterior ao jogo", "Ser prejudicado pe- 
los juizes", "Condicionamento físico inadequado" foram avaliadas com as respostas mais negativas. As situações "Cobrança de si mesmo para ganhar" e "O time adversário é o favorito" foram avaliadas com respostas mais positivas.

Na tabela 1 estão apresentados os valores da subtração pré e pós o protocolo experimental no dois grupos (GC e GA). Pode-se observar que o grupo GA obteve aumento no número de avaliações positivas comparado ao GC, diminuição no valor $\mathrm{O}$ e diminuição na resposta -1 e -3 , enquanto o GC obteve diminuição no valor -2.

Após multiplicação da subtração dos valores pré e pós o protocolo experimental em todas as situações propostas pelo TEF nos grupos GC e GA pode-se observar melhor a diminuição da avaliação negativa e aumento da avaliação positiva das situações geradoras de estresse dos participantes do grupo com alongamento comparados ao grupo controle (gráfico 2).

\section{DISCUSSÃO}

O futebol apresenta uma séria de situações geradoras de estresse. Os exercícios físicos, particularmente $o$ alongamento, podem contribuir para diminuir os efeitos negativos do estresse.

No presente estudo foram verificadas as avaliações das situações propostas pelo TEF. As situações "Dormir mal a noite anterior ao jogo", "Ser prejudicado pelos juizes" e "Condicionamento físico inadequado" foram avaliadas como as mais estressantes com avaliação negativa para os atletas. Sabe-se que o sono é fundamental para melhora no rendimento dos atletas e tal resultado corrobora com a literatura ${ }^{7}$, bem como condicionamento físico inadequado. Para o

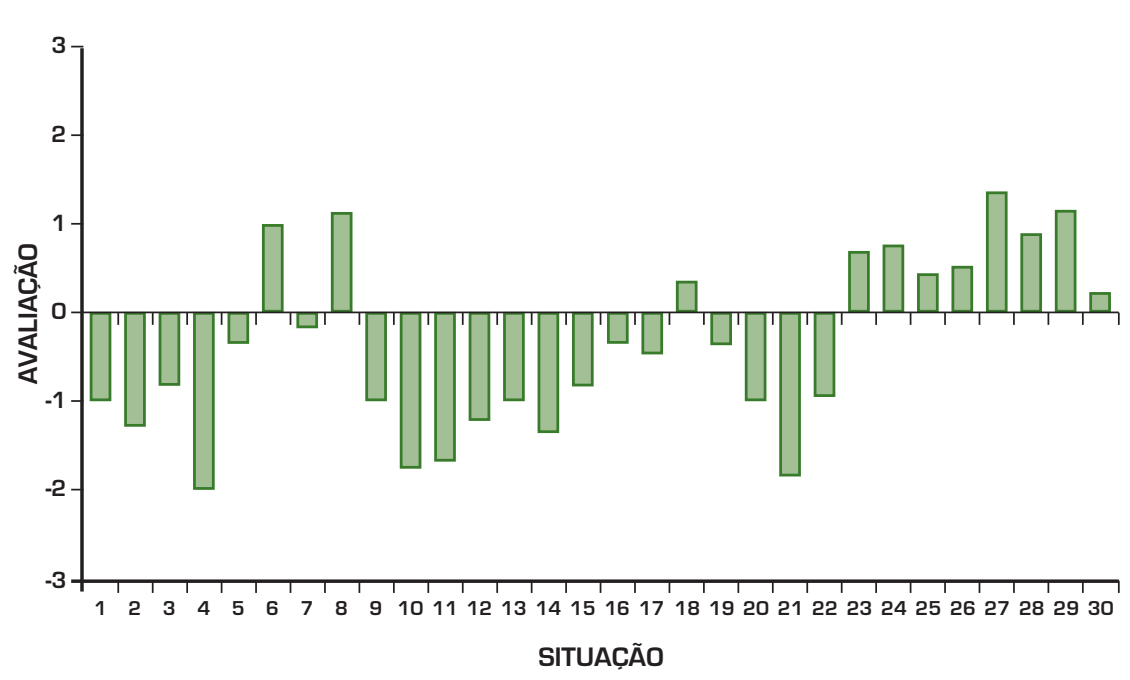

FIGURA 1

Médias das avaliações feitas pelos atletas nas determinadas situações propostas pelo TEF durante o protocolo experimental $(n=17)$.

\begin{tabular}{lccccccc} 
Valores & -3 & -2 & -1 & 0 & 1 & 2 & 3 \\
\hline GC & 5 & -13 & 9 & 7 & 2 & 0 & -7 \\
GA & 2 & 7 & -9 & -13 & 4 & 3 & 7 \\
\hline
\end{tabular}

TABELA1

Subtração dos valores pré e pós o protocolo experimental em todas as situações propostas pelo TEF nos grupos GC e GA ( $n=17)$.

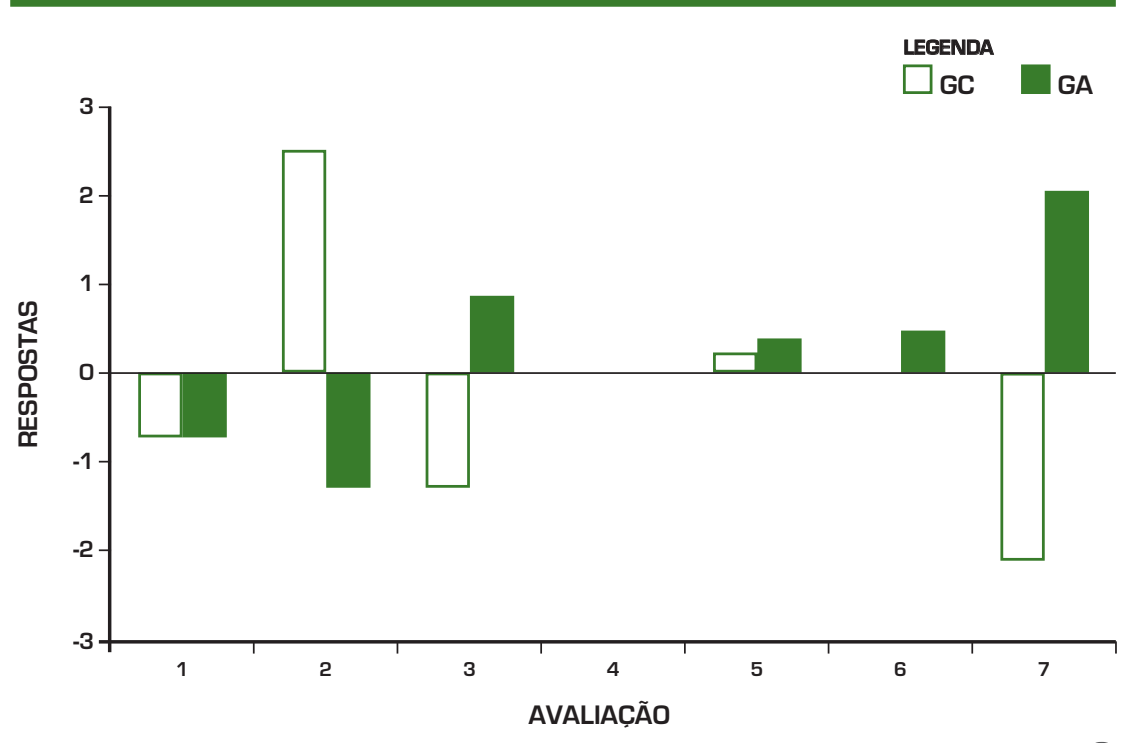

FIGURA?

Multiplicação da subtração dos valores pré e pós o protocolo experimental em todas as situações propostas pelo TEF nos grupos GC e GA ( $n=17)$. 
treinamento físico ainda é necessário respeitar a idade cronológica e maturidade biológica dos atletas de categoria de base ${ }^{8}$. Ser prejudicado pelo juiz também foi considerada uma situação muito negativa após a aplicação de outro questionário em outro grupo de atletas profissionais e amadores ${ }^{9}$.

As situações "Cobrança de si mesmo para ganhar" e "O time adversário é o favorito" foram avaliadas como as respostas mais positivas. Estas duas variáveis também foram consideradas motivantes em estudos prévios ${ }^{9,11}$.

O grupo controle demonstrou aumento do estresse no momento pré-competitivo, com aumento das análises negativas frente às situações. Isso mostra que a situação pré-competitiva é uma situação geradora de estresse, podendo haver prejuízo no desempenho do atleta se não houver nenhuma interferência. Este resultado está de acordo com os dados encontrados em estudo prévio encontrados na literatura que relatam alterações psicofisiológicas que podem interferir no desempenho do atleta durante a competição ${ }^{12,13}$.

A interferência do alongamento no GA obteve aumento no número de avaliações positivas comparado ao GC, diminuição no valor 0 e diminuição em duas das avaliações negativas (-1 e -3). Este resultado corrobora com outros estudos que verificaram a interferência da atividade física no estresse. Nunomura ${ }^{14}$ obteve como resultado melhoria significativa nos sintomas geradores de estresse após 12 meses de prática de atividade física, sugerindo a influência positiva da atividade física regular na atenuação do desencadeamento do processo de estresse entre os adultos. Valim ${ }^{4}$ demonstrou que a aplicação de uma sessão de alongamentos é uma intervenção eficaz na redução dos sintomas de estresse em jovens.
Baseado nos resultados encontrados neste trabalho pode-se concluir que a situação pré-competitiva modifica negativamente a avaliação dos atletas e que o alongamento interfere positivamente frente à avaliação das situações estressoras em jogadores de futebol juvenil na situação pré-competitiva.

\section{CORRESPONDÊNCIA}

José Alexandre Curiacos de Almeida Leme

Av. Armando Salles de Oliveira, 1574, ap. 72

Piracicaba, São Paulo, Brasil

Cep 13400-000

E-mail: zecuriacos@terra.com.br

\section{REFERÊNCIAS}

1. Brandão R (2000). Fatores de estresse em jogadores de futebol profissional. Tese (Doutorado) Faculdade de Educação Física, Universidade Estadual de Campinas, Campinas.

2. Samulski D; Chagas MH (1996). Análise do estresse psíquico na competição em jogadores de futebol de campo das categorias juvenil e junior. Rev Assoc Prof Educ Fis Lond 11: 3-11.

3. Bidutte LC; Azzi RG; Raposo JV; Almeida L (2006). Agressividade em equipas de futebol. Motricidade 2 (1): 13-22.

4. Valim PC; Bergamaschi EC; Volp CM; Deutsch $S$ (2003). Redução de Estresse pelo Alongamento: a Preferência Musical Pode Influenciar? Motriz v. 8, n. 2, pp. 51-56.

5. Phillips AC; Burns VE; Lord JM (2007). Stress and exercise: Getting the balance right for aging immunity. Exerc Sport Sci Rev. 35(1):35-9.
6. Hassemen P; Koivula N; Uutela A (2000). Physical exercise and psychological well-being: A population study in Finland. Preventative Medicine 30: 17-25.

7. DeRose DJ (1998). Lista de sintomas de "stress" pré-competitivo infanto-juvenil: elaboração e validação de um instrumento. Rev Paul Educ Fís, 12(2): 126-33.

8. Villar R; Zühl CA (2006). Efeitos da idadecronológica e da maturação biológica sobre a aptidão física em praticantes de futebol de 13 a 17 anos. Motricidade 2 (2): 69-79.

9. Corrêa DKA; Alchieri JC; Duarte LRS; Neves M (2002). Excelência na produtividade: a performance dos jogadores de futebol profissional Psicol. Reflex. Crit. 15(2): 447-460. 10. Sanches MAB; Casal HMV; Brandão MRF (2004). Fatores de estresse no futebol Lecturas educacion fisica y deportes 10(73) 1-18.

11. Chagas MH. Análise do estresse psíquico na competição em jogadores de futebol de campo das categorias juvenil e júnior. Belo Horizonte: Escola de Educação Física da UFMG, Dissertação de mestrado em Ciências do Esporte, 1995.

12. Samulski D; Noce F (2002). Perfil psicológico de atletas paraolímpicos brasileiros Rev Bras Med Esporte. 8(4): 157-166.

13. Girardello RJR [2004). A relação entre o cortisol sanguíneo e o estresse pré-competitivo em lutadores de caratê de alto rendimento. Dissertação de Mestrado em Educação Física. Universidade Federal do Paraná.

14. Nunomura M; Teixeira LAC; Caruso MRF (2004). Nível de estresse em adultos após 12 meses de prática regular de atividade física. Rev Mack Educ Fís Esp. 3(3): 125-134. 